\title{
Quantifying the Redistribution of Mass in Galactic Disks due to Bars
}

\author{
Patricia Sánchez-Blázquez ${ }^{1}$, Isabel Pérez ${ }^{2}$ and Pierre Ocvirk ${ }^{3}$ \\ ${ }^{1}$ Departamento de Física Teórica, Universidad Autónoma de Madrid, Cantoblanco 28049, \\ Spain \\ email: p.sanchezblazquez@uam.es \\ ${ }^{2}$ Departamento de Física Teórica, Universidad de Granada,18071, Spain \\ ${ }^{3}$ Observatoire astronomique, 6700, Strasbourgh, France
}

\begin{abstract}
Numerical simulations have shown that strong gravitational torque by non-axisymmetric components induce evolutionary processes such as redistribution of mass and angular momentum in the galactic disks and consequent change of chemical abundance profiles. If we hope to understand chemical evolution gradients and their evolution we must understand the secular processes and re-arrangement of material by non-axisymmetric components and viceversa. The most obvious of these aforementioned non-axisymmetric components are bars - at least $2 / 3$ of spiral galaxies host a bar, and possibly all disk galaxies have hosted a bar at some point in their evolution. While observationally it has been found that barred galaxies have shallower gas-phase metallicity gradients than non-barred galaxies, a complementary analysis of the stellar abundance profiles has not yet been undertaken. This is unfortunate because the study of both gas and stars is important in providing a complete picture, as the two components undergo (and suffer from) very different evolutionary process. We present here a pilot study of the gas and stellar metallicity and age distributions in a sample of barred and non-barred galaxies using $2 \mathrm{D}$ spectroscopic observations. We found that the majority of the stellar mass in our sample is composed of old ( $\sim 10 \mathrm{Gyr})$ stars. This is true in the bulge and the disc region, even beyond two disc scalelengths. In the disc region, a larger fraction of young stars is present in the external parts of the disc compared with the inner disc. The disc growth is, therefore, compatible with a moderate inside-out formation scenario, where the luminosity-weighted age changes from $\sim 10$ Gyrs in the centre, to $\sim 4$ Gyrs at two disc scalelengths, depending upon the galaxy. However, the presence of substructure, like star forming rings, can produce stellar population trends that are not directly related with the growing of the disc but to the bar potential. In the disc region, the metallicity gradient always decrease with the radius. In the bulge region this is not always true and we find inverse metallicity gradients in several galaxies.
\end{abstract}

Keywords. galaxies:evolution, galaxies:stellar content, galaxies:spiral, galaxies:formation, galaxies: abundances

\section{Introduction}

During disc assembly, secular evolution must have played a role in shaping the structure of disc galaxies as we see them at $\mathrm{z}=0$. Non-axisymmetric instabilities, particularly bars, drive a substantial redistribution of mass and angular momentum in the disc (e.g., Pfenniger \& Friedli 1991; Debattista et al. 2006). Since bars appear to affect strongly the overall dynamics and evolution of galaxies, it is reasonable to presume that they also play a significant role in shaping galactic chemical evolution, since mixing by global gas flows will clearly change abundance profiles in the disc (see Roškar et al. 2008b; Sánchez-Blázquez et al. 2009); for example, numerical simulations (Friedli, Benz \& Kennicutt 1994) predict that the stellar abundance gradient in disc galaxies is flattened by macroscopic mixing induced by the bar. The slope of the abundance gradients in both 
Table 1. Galaxies analysed in this pilot study. The name, morphological type and absolute magnitude in the B-band are provided.

\begin{tabular}{l|c|c|c|c|c|}
\hline Gal & Typ & Mb & Gal & Typ & Mb \\
\hline NGC628 & SA(s)c & -19.9 & NGC1672 & SBb & -20.7 \\
NGC1058 & SA(rs)c & -18.3 & NGC3184 & SAB(rs)cd & -19.9 \\
NGC1358 & S0B(r) & -20.9 & NGC3310 & SAB(r)bc & -20.1 \\
NGC1365 & SBb & -21.7 & NGC1637 & SAB(rs)c & -18.9 \\
NGC1433 & SBa(r) & -19.9 & & & \\
\hline
\end{tabular}

the stellar and gaseous component are reduced in a few dynamical timescales by more than $50 \%$. Several studies have found that barred galaxies have a shallower gas-phase metallicity gradient than non-barred ones (e.g.,Vila-Costas \& Edmunds 1992; Zaritsky et al. 1994). However, an equivalent study for the stellar abundances remains to be done. Studies of the gas-phase abundances provide with a present-day snapshot of the interstellar medium abundances. On the other hand, the study of the stellar ages and metallicities give us 'archaeological' clues as to the chemical and dynamical evolution of the galactic structures. It is very important to study both because gas and stars suffer from very different evolutionary processes; the gas is mainly dominated by the gravitational torque of the non-axisymmetric mass component, while the evolution of the stellar component is mainly affected by different orbital mixing. Here we present a preliminary study of the metallicity gradient in the stellar component of a sample of disk galaxies using 2D spectruscopy from the PINGS survey (Rosales-Ortega et al. 2010) and long-slit spectroscopy from the GEMINI telescope (Sánchez-Blázquez et al. 2010, in preparation). The details of the observations and data reduction are provided in these two studies. This pilot study has been performed with 9 face-on galaxies of spectra types from Sa to Sc (see Table 1). The subsample presented here is biased toward barred-types but the complete sample will include equal numbers of barred and non-barred galaxies.

\section{Stellar Population gradients}

We use here STECKMAP (STEllar Content and Kinematics via Maximum a Posteriori, Ocvirk et al. 2006a,b), along with the stellar population models by Vazdekis et al. (2010), spanning an age range $10^{8}-1.7 .10^{10}$ yr divided in 30 logarithmic age bins, and a metallicity range $[\mathrm{Z} / \mathrm{H}]=[+0.2,-1.3]$. STECKMAP is a Bayesian method that simultaneously recovers the kinematic and stellar population properties via a maximum a posteriori algorithm. We derive star formation histories using this algorithm and obtain a value for the metallicity weighted in both, mass and stellar luminosity. Figure 1 and 2 shows the mean metallicity and age gradients obtained this way for our sample of galaxies. The gradients represents the average, at each radius, weighting with the mass and the light of the stars.

It can be seen in the figures that galaxies show a variety of behaviours in the change of the stellar metallicity with radius. The majority of galaxies show standard gradients, with the central parts more metal rich than the externals. However, some galaxies (NGC 1365, NGC 1637, NGC 3184) present a positive metallicity gradient with nucleus depleted in metals compared to other galaxies of similar magnitude and spectral type. What it is interesting in these galaxies is that, despite they have the lowest metallicities in the bulge region, they show the highest stellar abundances in the disk regions when compared with similar galaxies. It has been claimed recently that this kind of 'inverse gradient' with the central regions having a lower metalicity than the rest of the galaxy is a clear signature of accretion of primordial gas (Cresci et al. 2010). However, minor mergers can also produce this type of gradients. Recently, Elmegreen, Galliano \& Alloin (2009) have studied the gas 

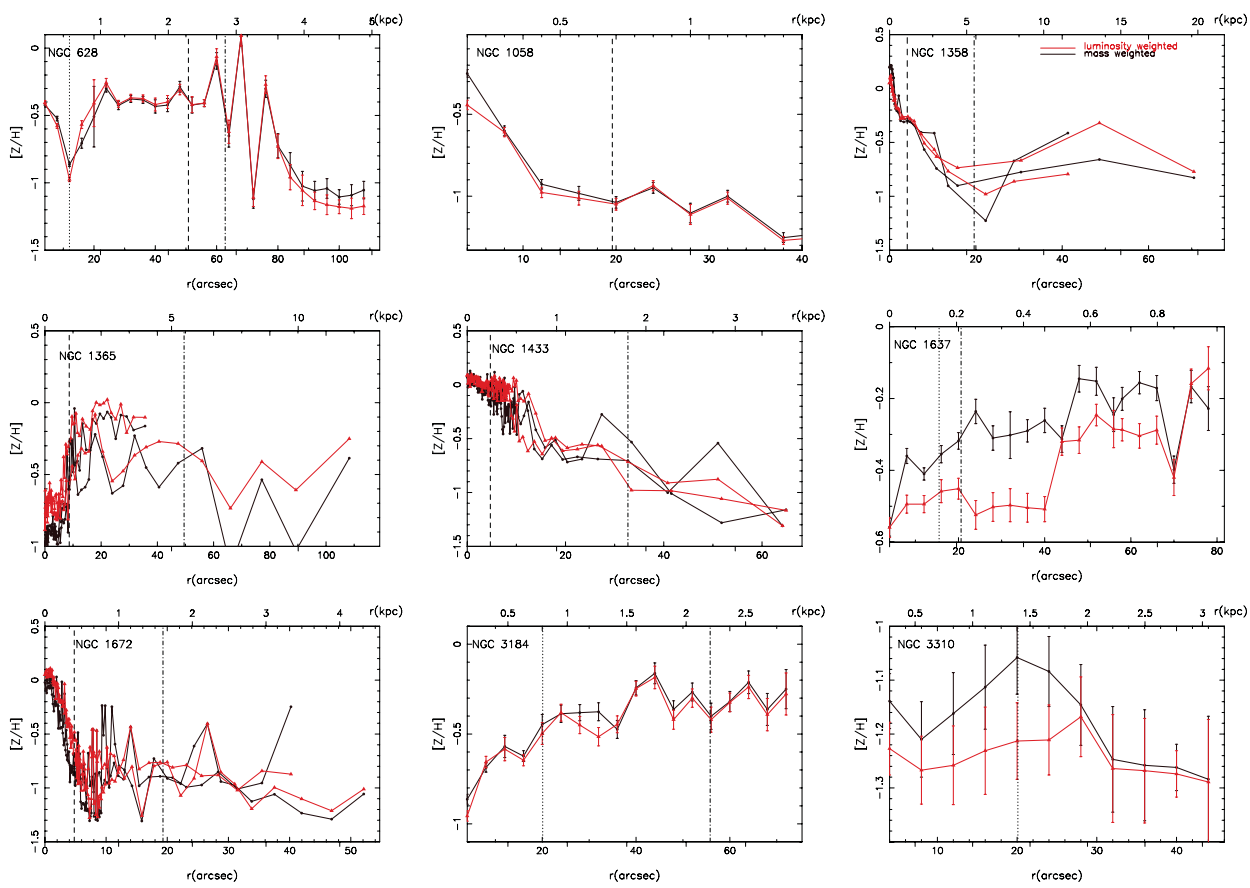

Figure 1. Luminosity (red lines) and mass-weighted (black lines) metallicity gradients for our sample of galaxies.
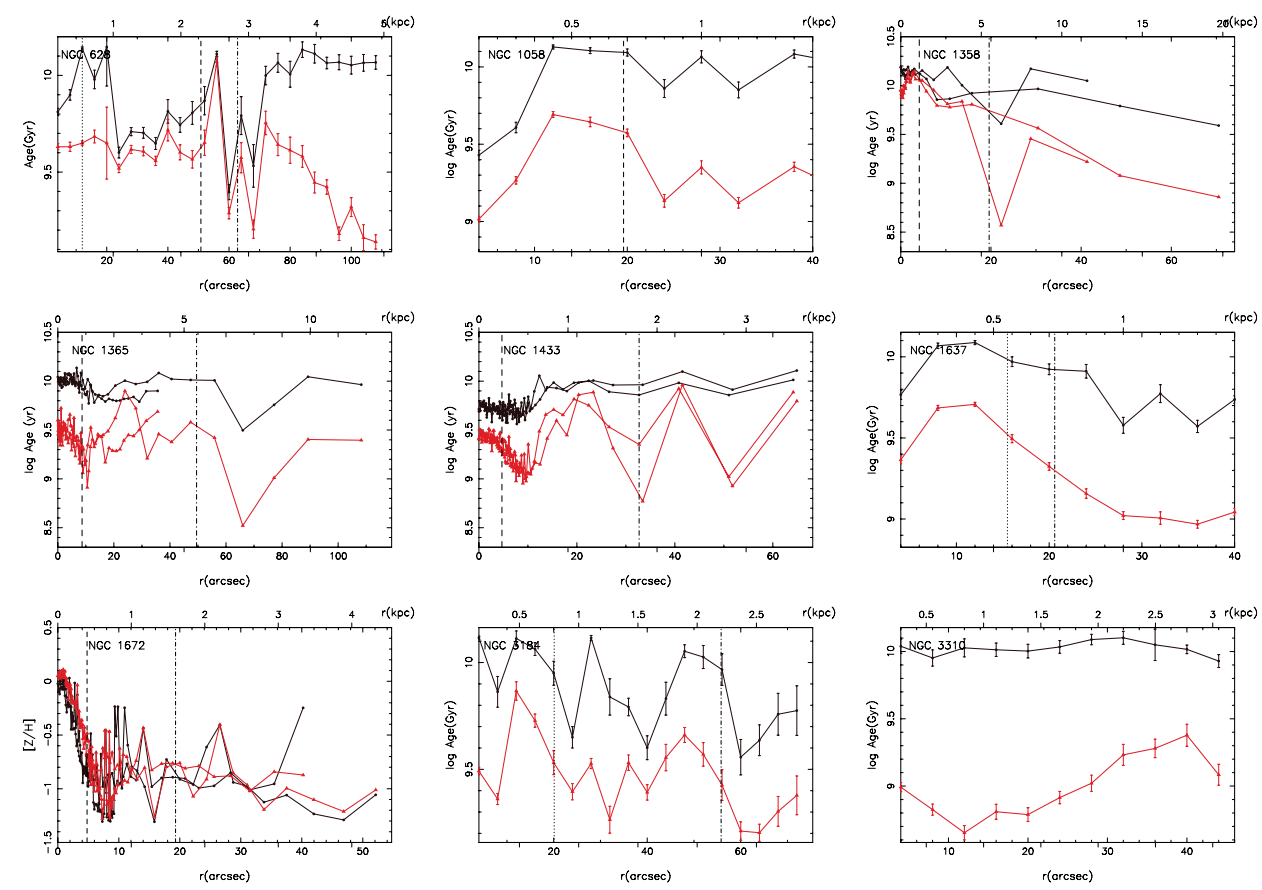

Figure 2. Luminosity (red lines) and mass-weighted (black lines) age or our sample of galaxies. 

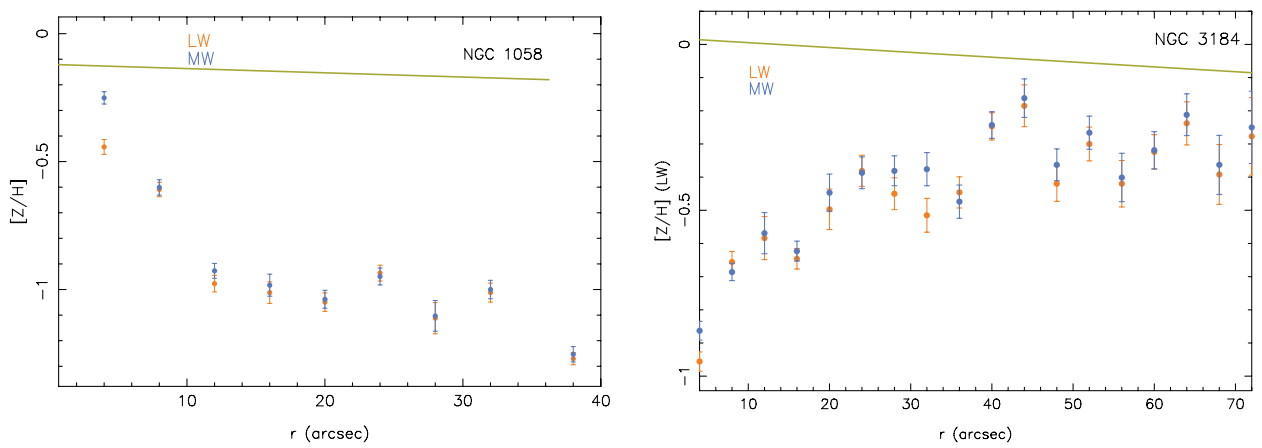

Figure 3. Comparison of the stellar and gas-phase metallicities of the unbarred galaxies NGC 1058 and the barred NGC 3184. The orange and blue symbols represent the luminosity and mass-weighted stellar values respectively, while the gas-phase metallicity gradient is depicted with a green line.

dynamic in NGC 1365 finding strong gas flows from the outskirts of the galaxy inwards at a rate of $\sim \mathrm{M}_{\odot} \mathrm{yr}^{-1}$ near the ILR. This flows can be the reason of the low metallicities in the nuclear regions.

All the galaxies are dominated in mass by an old stellar population (in the bulge and in the disc). The mass-weighted age gradients in the disc regions are always quite flat with a mean age around 10-12 Gyr. The luminosity weighted age gradient is either flat or slightly negative (at 2 scale-lengths, the age decrease is 4 Gyr for NGC 1672 and $\sim 4.7$ Gyr for NGC 1358, for example), consistent with an inside-out formation of the disc.

For some galaxies, we have been able to compare the stellar and gas-phase metallicity gradients. Figure 3 shows an example for an unbarred (NGC 1058) and a barred (NGC 3184) galaxy. In the future, we will extend this comparison to the rest of the PINGS sample and compare with appropiate models to derive the degree of radial mixing in the disc due to the bar presence.

\section{References}

Berentzen, I., Shlosman, I., Martínez-Valpuesta, I., Heller, C. H., 2007, ApJ, 666, 189

Bournaud F., Combes F., \& Semelin B., 2005, MNRAS, 364, 18

Cresci, G., Mannucci, F., Maiolino, R., Marconi, A., Gnerucci, A., \& Magrini, L., 2010, Nature, 30,490

Debattista, V. P., Mayer, L., Carollo, C. M., Moore, B., Wadsley, J., \& Quinn, T., 2006, ApJ, 645,209

Friedli, D., Benz, W., \& Kennicutt, R., 1994, ApJ, 430, 105

Gadotti, D., 2008, in Chaos in Astronomy, Ed. G. Contopulos \& P.A. Patsis.

Ocvirk, P., Pichon, C., Lançon, A., \& Thébaut, E., 2006, MNRAS, 365, 46

Ocvirk, P., Pichon, C., Lançon, A., \& Thiébaut, E., 2006, MNRAS, 365, 74

Pérez, I., Sánchez-Blázquez, P., \& Zurita, A.,2009, A\&A, 495, 775

Pfenniger, D. \& Friedli, D., 1991, A\&\&A, 252, 75

Rosales-Ortega, F. F., Kennicutt, R. C., Sánchez, S. F., Díaz A. I., Pasquali, A., Johnson, B. D., \& Hao, C. N., 2010, MNRAS, 405,735

Roškar, R., Debattista, V. P., Quinn, T. R., Stinson, G. S., \& Wadsley, J., 2008, ApJ, 648, 79

Sánchez-Blázquez, P., Courty, S., Gibson, B. K., \& Brook, C. B., 2009, MNRAS, 398, 591

Shen, J. \& Sellwood, J. A., 2004, ApJ, 604, 614

Vazdekis, A., Sánchez-Blázquez, P., Falcón-Barroso, J., Cenarro, A. J., Beasley, M. A., Cardiel, N., Gorgas, J., \& Peletier, R. F., 2010, MNRAS, 404, 1639

Vila-Costas, M. B. \& Edmunds, M. G., 1992, MNRAS, 259, 121

Zaritsky, D., Kennicutt, R. C., Jr; \& Huchra, J. P., 1994, ApJ, 420, 87 